\title{
ON SUM-FREE SUBSEQUENCES ${ }^{1}$
}

\author{
DAVID G. CANTOR
}

\begin{abstract}
A sequence of real numbers is said to be sum-free if no number of the sequence is the sum of distinct elements of the same sequence. In this paper we show that a sequence $S$ of $n$ positive real numbers has a sum-free subsequence containing at least $(2 n)^{1 / 2}-\log _{2}(4 n)$ elements.
\end{abstract}

Choi [1], studying a problem of Erdös [2], has proven that a sequence of $n$ positive real numbers has a sum-free subsequence of $\geqq(36 / 35) n^{1 / 2}$ elements. The purpose of this paper is to show that the constant $36 / 35$ can be improved to $2^{1 / 2}-\varepsilon$. In fact, more precisely, we have

THEOREM. A sequence $S$ of $n$ positive real numbers has a sum-free subsequence containing $\geqq(2 n)^{1 / 2}-\log _{2}(4 n)$ elements.

Proof. Let $a_{1}$ be the least element of $S$; let $a_{2}$ be the least element of $S$ which is $\geqq 2 a_{1}$, and inductively choose $a_{i+1}$ to be the least element of $S$ which is $\geqq 2 a_{i}$. This yields a finite sequence $a_{1}<a_{2}<\cdots<a_{m}$ and every element of $S$ is $<2 a_{m}$. For $1 \leqq i \leqq m-1$, let $T_{i}$ be the subsequence of $S$ consisting of those elements of $S$ which are $\geqq a_{i}$ and $<a_{i+1}$; let $T_{m}$ be the subsequence of $S$ consisting of those elements of $S$ which are $\geqq a_{m}$. Denote by $t_{i}$ the cardinality of $T_{i}$. Then $S$ is the disjoint union of the $T_{i}$ and $\sum_{i=1}^{m} t_{i}=n$. Now

or

$$
\sum_{i=1}^{m}\left(t_{i}+m-i\right)=n+\frac{m(m-1)}{2}
$$

$$
\frac{1}{m} \sum_{i=1}^{m}\left(t_{i}+m-i\right)=\frac{n}{m}+\frac{m}{2}-\frac{1}{2} .
$$

Minimizing over $m$ shows that $(1 / m) \sum_{i=1}^{m}\left(t_{i}+m-i\right) \geqq(2 n)^{1 / 2}-\frac{1}{2}$. Thus there is an index $j$ such that $t_{j}+m-j \geqq(2 n)^{1 / 2}-\frac{1}{2}$. Define $k=j+1+\left[\log _{2} j\right]$. (Here and throughout $[x]$ denotes the least integer $\geqq x$ ). Put $V=$ $\left\{a_{k}, a_{k+1}, \cdots, a_{m}\right\} ; V$ is a sum-free sequence because its elements grow so rapidly that each element of $V$ is greater than the sum of all smaller

Received by the editors April 23, 1973.

AMS (MOS) subject classifications (1970). Primary $10 \mathrm{~L} 10$.

${ }^{1}$ The preparation of this paper was supported in part by NSF grant GP-3358X.

(C) American Mathematical Society 1974 
elements of $V$. The sequence $T_{j}$ is sum-free because each element of $T_{j}$ is $\geqq a_{j}$ and $<2 a_{j}$, and as a result the sum of any two elements of $T_{j}$ is greater than any element of $T_{j}$. Finally,

the sum of all the elements of $T_{j}<t_{j} \cdot 2 a_{j}$

$$
\begin{aligned}
& =2^{\left(1+\log _{2} t_{j}\right)} a_{j} \leqq 2^{1+\left\lceil\log _{2} t_{j}\right\rceil} a_{j} \\
& \leqq a_{j+1+\left\lceil\log _{2} t_{j}\right\rceil}=a_{k} \text { if } k \leqq m .
\end{aligned}
$$

It is immediate that if $a_{i} \in V$, then $a_{i}$ is greater than the sum of all smaller elements of $T_{j} \cup V$ and hence $T_{j} \cup V$ is a sum-free subsequence of $S$ with

$$
\begin{aligned}
\text { number of elements } & \geqq t_{j}+m-k+1=t_{j}+m-j-\left[\log _{2} j\right] \\
& \geqq(2 n)^{1 / 2}-\frac{1}{2}-\log _{2}(2 j) \geqq(2 n)^{1 / 2}-\frac{1}{2}-\log _{2}(2 n) \\
& \geqq(2 n)^{1 / 2}-\log _{2}(4 n) .
\end{aligned}
$$

\section{REFERENCES}

1. S. Choi, The largest sum-free subsequence from a sequence of $n$ numbers, Proc. Amer. Math. Soc. 39 (1973), 42-44.

2. P. Erdös, Extremal problems in number theory, Proc. Sympos. Pure Math., vol. 8, Amer. Math. Soc., Providence, R.I., 1965, pp. 181-189. MR 30 \#4740.

Department of Mathematics, University of California, los Angeles, CaliFORNIA 90024 